\title{
Development of Universal Exergy Analysis to the Selection of Cryogenic Cooling System
}

\author{
S. V. Zaika , D. A. Uglanov, S. S. Dostovalova \\ Samara University, Heat engineering and heat engines Department, Samara, Russia
}

\begin{abstract}
Efficiency is one of the most important parameters that apply to the cooling system. This paper describes a development of universal methodology to evaluate the effectiveness of cryogenic cooling systems. The problem of the lack of a universal method of comparison of cryogenic cooling systems was solved. This was achieved by creating a universal method of exergy analysis. In addition, as an example were examined three of the cooling system, which applies the universal exergy analysis. Basic universal parameters that characterize cooling system level of sophistication were defined, as well as main calculated. The result of this study is a new universal method of exergy analysis, which can be used to select a cryogenic cooling system.
\end{abstract}

\section{Introduction}

The most important requirement for many energy projects is providing a cooling of IR-receivers, elements of optical systems and other devices to be installed on power plant to cryogenic temperature. When choosing an cryogenic cooling system one should take into account not only the energy waste, but also waste that arise due to the cooling system organizational management, such as: acquisition and transportation of the working fluid waste, the heat flows waste, waste due to equipment imperfection [1].

Evaluation of any thermodynamic system power resources, as well as energy transformation, should take into account the influence of environmental parameters. Therefore, using the concept of energy as a common measure of the matter motion in considered system is insufficient when solving engineering problems. The technology development makes us consider the fact that not any kind of energy and not under any conditions can be entirely suitable for practical use. Technical value of energy depends on its kind and parameters, as well as on the environment parameters.

From the standpoint of technical feasibility any kind of energy value is determined not only by the energy amount, but also by the extent to which it can be used in set conditions, i.e. transformed into other kinds of energy. It is important to study transformed energy waste when performing thermodynamic analysis of technical processes.

Mechanical or electrical energy, fully converted into another form of energy, can be taken as the usage measure of any kind of energy. Therefore, if a certain amount of energy can be transformed into electrical or mechanical energy in the set conditions, that fully guarantees the possibility of complete transformation of this amount of energy in any other kind of organized energy. Conditions of such transformation are determined by the second law of thermodynamics.

\section{The basic equation of exergy}

In the light of the foregoing, it is necessary to introduce a common measure for the energy resources capable of transformation into other forms of the organized energy when constant parameters are interacting with the environment. It is clear that the ultimate energy transformation can only occur when all the processes within the system, as well as its interaction with the environment are reversible.

Forenamed measure of transformed energy resources of the system is referred to as an exergy of the system. In the mentioned state it is measured by the amount of mechanical, or other fully transformed energy, that can be obtained from this system due to its reversible transition from this state to the state of equilibrium with the environment.

It directly follows from the first and second laws of thermodynamics that in each state system's exergy, as well as energy, has a certain fixed value.

Exergy of the system, existing in the environment of constant parameters, remains unchanged only in case of reverse processes, occuring within it, as well as in case of interacting with the environment. If any of these interactions are irreversible, exergy of the system decreases accordingly.

This basic property of exergy allows us to use it as the measure of the process' reversibility. The difference between total amount of the exergy input of the system $\left(E_{\text {in }}\right)$ and amount of the exergy output $\left(E_{\text {out }}\right)$, determines 
the total value of waste due to the system irreversibility by eqn (1):

$$
\sum D=\sum E_{\text {in }}-\sum E_{\text {out }}>0
$$

The exergy balance equation can be written expressing the useful work for a general process as depending on the useful work for the corresponding reversible process and the exergy destruction term. However, this can be made only if the terms (other than the exergy destruction term) on the exergy balance equation for the general process lead to the same result as the terms on the exergy balance equation for the corresponding reversible process [2].

The exergy loss is similar to the entropy, which increase in a closed system also reflects waste due to system irreversibility. However, the practical advantage of the exergy concept is that it gives a decrease in the value of the transformed energy waste right away and allows you to compare waste value to the available amount of transformed energy (i.e. to find both absolute and relative waste values). The ratio of the exergy output of the system $\left(E_{\text {out }}\right)$ for the evaluation of exergy input $\left(E_{\text {in }}\right)$ is represented by the efficiency - the exergy efficiency, which characterizes the process degree of approximation to the ideal is determined by the eqn (2):

$$
\eta=\frac{\sum E_{\text {out }}}{\sum E_{\text {in }}}=\frac{\sum E_{\text {out }}-\sum D}{\sum E_{\text {in }}}=1-\frac{\sum D}{\sum E_{\text {in }}}
$$

In ideal process $\eta=1$, in real process $\eta<1$.

Waste (D) can be divided into two groups in terms of their distribution:

- internal, associated with the irreversibility of the processes, occurring within the system. They are usually denoted by the letter $\mathrm{i}\left(\mathrm{D}_{\mathrm{i}}\right)$;

- external, related to the terms of system interaction with the environment and other sources and receivers of energy. They are denoted by the letter e $\left(D_{e}\right)$.

In heat transformers and refrigeration units, in particular, waste due to the throttling, hydraulic resistance, friction in machines, heat and mass transfer at finite temperature and concentration head, etc. may serve as an example of internal waste. External waste include those associated with temperature difference between the working fluid and the coolant, as well as waste through the heat insulation. Waste of the products, leaving the plant, which exergy is not consumed also belong to this group, e.g. heated cooling water, waste nitrogen in oxygen plants, and others.

Internal and external waste separation can be easily produced by the same formula. If it contains assumed exergy values, taken according to the parameters of the plant, the obtained value $\mathrm{D}_{\mathrm{i}}$ will correspond only to the internal waste. If the equation contains exergy values either given to or received from the external sources and receivers of energy, then $\mathrm{D}$ includes both external and internal waste. Consistently applying the equation to the individual elements of the plant, it is easy to determine the distribution of the plant internal waste.

The energy balance of this system is determined by the eqn (3):

$$
(L+E+Q+I)_{\text {in }}=(L+E+Q+I)_{o u t}
$$

The energy balance of the system is determined by the equation (1), which shows that in real processes part of the energy is converted into inoperative form.

By the exergy the energy efficiency is meant, i.e. the amount of work_operation that can be obtained with the set energy as a result of reversible interaction with the environment.

In case of mechanical and electrical kinds of energy, exergy and energy values are equivalent, as in an ideal process these kinds of energy can be transformed into work operation entirely. For other energy kinds, such as the heat flux, exergy value is determined by the amount of energy that can be transformed into work operation in the perfect tune [3].

The first law of thermodynamics imposes no restrictions on the transition of one kind of energy, it is only important that the amount of total remain unchanged; the first law of thermodynamics imposes certain restrictions on the energy transformation.

For the exergy balance of the system it is necessary to use the method of determining the exergy of each kind of energy.

\section{Exergy analysis of major cryogenic cooling systems}

It is necessary to consider the most common system before proceeding with the development of calculation methods.

Exergy analysis gives the presentation of a system relative to its best performance. In addition, the exergy destructed can react with its surrounding and harm environment processes [4].

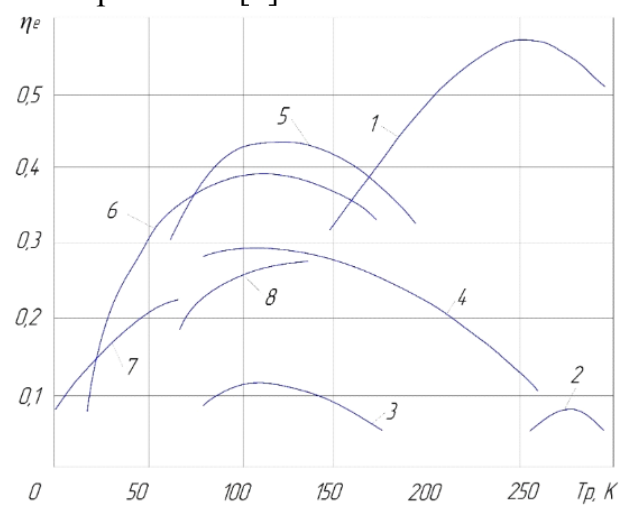

Figure 1. Low-temperature plants' exergy efficiency relation to cryostatting temperature.

Figure 1 shows the exergy efficiency relation on cryostatting temperature for some cycles and patterns of cryogenic plants. The graph shows that for each cooling method there is a relatively narrow temperature range, within which a high performance of the system remains. Maximum efficiency in the temperature range of 70-150 $\mathrm{K}$ belongs to the gas cryogenic machines, running on the Stirling cycle (curves 5,6), minimum - to the throttle system, in which nitrogen is used as a refrigerant (curve 3). The use of thermoelectric coolers (curve 2) in microcryogenic systems is limited by the temperature 
conditions. Throttle cryogenic systems are most easily amenable to miniaturization, but have the lowest efficiency in comparison with other types of closed systems due to the imperfections of the throttling process and small isothermal efficiency. However, the effectiveness of throttle microcryogenic systems can be significantly increased by the coolants' thermal properties improvement [5].

In case of miniaturization, Stirling cycle systems retain the highest efficiency as compared with other cycles and cooling methods.

1 - steam compressor units; 2 - thermoelectric coolers; 3 throttle system with nitrogen as a refrigerant; 4 - regenerative systems with turbomachines; 5,6 - horizontal forging machine working on Stirling cycle; 7 - expanders; 8 - throttle system using gas mixtures.

\subsection{Thermoelectric cooling system}

Thermoelectric cooling system (electronic refrigerator) is optimal device for receiver of radiative energy. Electronic refrigerators have several advantages compared with other cooling systems, if the receiver is to be cooled to $180 \mathrm{~K}$. They have low weight and dimensions, low energy consumption, high operational reliability.

Electronic refrigerators have elements which consist of two series-connected semiconductor branches with electronic conduction (n) and hole conduction (p). All thermocouples are connected in series or in parallel to form a thermopile (Figure 2) [6].

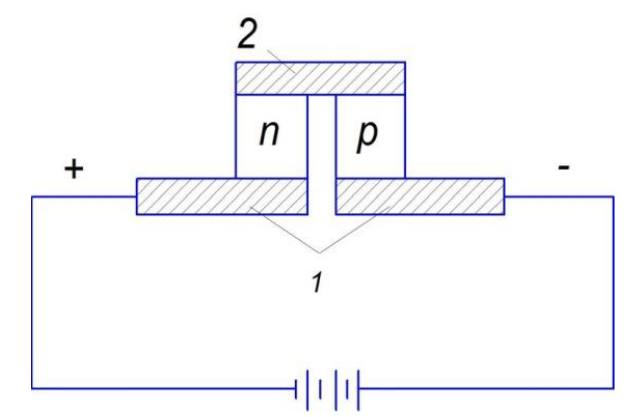

Figure 2. Elementary thermopile.

We need to use multi-stage thermopile (thermoelectric module) to provide the required temperature conditions (Figure 3). Selection standard thermoelectric modules depend on at least three parameters. This is temperature of hot and cold side of the module as well as the heat load that should be removed from the cold side.

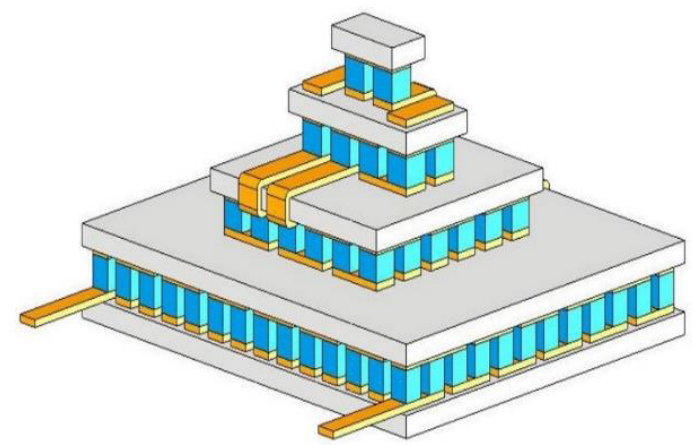

Figure 3. Four-stage thermoelectric module

\subsection{Throttle cooling system}

Outer contour of this type cooling system includes the following devices: throttle, tank for storing high-pressure gas, filter-adsorber (Figure 4).

According to calculations, using nitrogen as a refrigerant for cooling in the temperature range $200-250$ $\mathrm{K}$ and obtain cooling capacity of about $350 \mathrm{~W}$, it is necessary to provide the gas flow rate of about $15 \mathrm{~kg} / \mathrm{h}$.

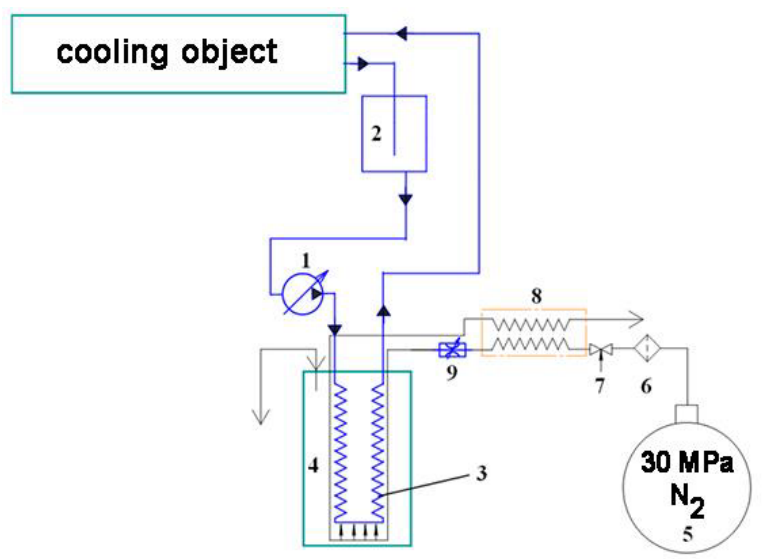

Figure 4. Throttle cooling system.

1 - pump; 2 - a broad tank; 3 - heat exchanger; 4 - an external coolant tank; 5 - a cylinder for storing high-pressure gas; 6 filter-adsorber; 7- flow control valve; 8 - exchanger of throttle system; 9 - throttle

Practical implementation of such a system makes it possible to cool not only the laser, but infrared receivers that are part of the complex laser.

\subsection{Refrigerating machines working on Stirling cycle}

Refrigerating machines working on Stirling cycle most widely used to provide the required temperature conditions. They have a high efficiency and very compact.

In a closed cycle Stirling working fluid is involved in a cyclic process consisting of four stages (two isochor and two isotherms).

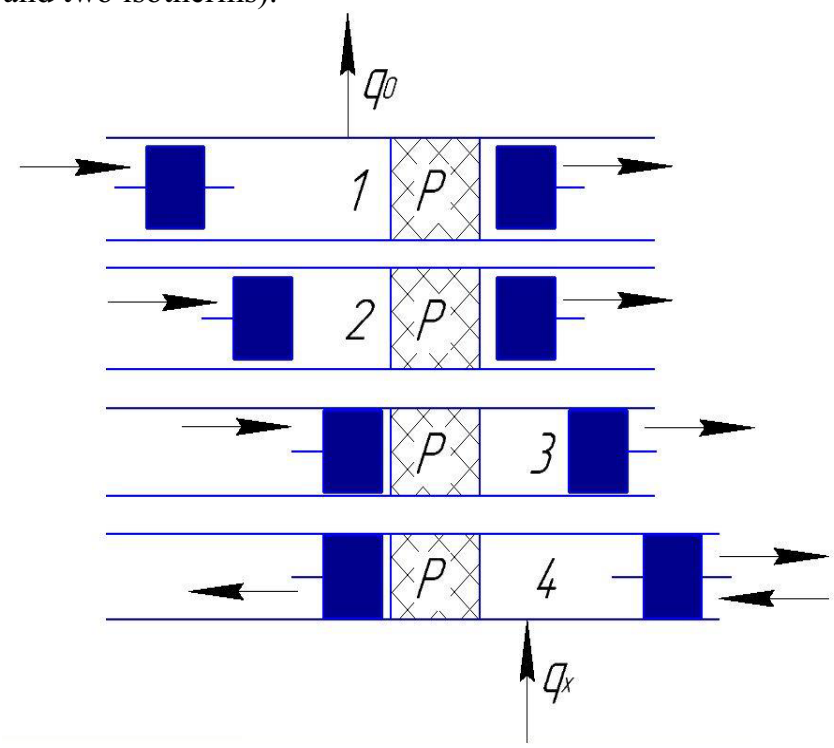

Figure 5. Reverse (refrigeration) cycle Stirling 
Heat is removed from the cycle into the environment occur at a temperature $\mathrm{T}_{0}$ in the compression. Heat is removed from the cooling body is passed into the cycle at the temperature $\mathrm{T}$ during expansion. The regeneration of the heat in cycle is carried out in the process $\mathrm{V}=$ const (Figure 5).

The work of compression (1-2) is determined by eqn:

$$
l_{c \varkappa}=l_{1-2}=R T_{0} \ln \frac{v_{2}-b}{v_{1}-b}-a\left(\frac{1}{v_{1}}-\frac{1}{v_{2}}\right)
$$

The work of expansion (3-4) is determined by the eqn:

$$
l_{p}=l_{3-4}=R T \ln \frac{v_{1}-b}{v_{2}-b}-a\left(\frac{1}{v_{2}}-\frac{1}{v_{1}}\right)
$$

The amount of heat taken away to the environment is determined by the eqn:

$$
q_{o c}=R T_{0} \ln \left[\frac{\left(v_{2}-b\right)}{\left(v_{1}-b\right)}\right]
$$

The amount of heat reported to the working fluid in the expansion process is determined by the eqn:

$$
q=R T \ln \left[\frac{\left(v_{1}-b\right)}{\left(v_{2}-b\right)}\right]
$$

Refrigeration Stirling cycle efficiency is determined by the eqn:

$$
\varepsilon_{S t r}=\frac{q}{l_{\Sigma}}=\frac{T}{\left(T_{0}-T\right)}=\varepsilon_{C}
$$

The change in internal energy in the compression (1-2) is determined by the eqn:

$$
\Delta u_{1-2}=\Delta u_{T_{0}}=u_{1}-u_{2}=a\left(\frac{1}{v_{2}}-\frac{1}{v_{1}}\right)
$$

The cooling capacity of the ideal machine Stirling with the real body work of expansion is determined by the eqn:

$$
q=l_{p}-\Delta u_{T_{0}}
$$

The fundamental way to implement the reverse Stirling cycle in a piston machine illustrated in Figure 5. Two pistons move in cylinder intermittently utlom phase shift $\beta$. In the working space between the piston positioned regenerator $\mathrm{P}$ which divides the working chamber into two parts - warm and cold. Sometimes they are conventionally called cavities of compression and expansion. In the warm cavity maintained at a constant temperature $T_{0}$ by removing heat qos refrigerated. The cold temperature of the cavity $\mathrm{X}$ is constant (due to the heat supply through the cooler q).

The above graph shows: 1) complex and different dependence of the efficiency $\eta_{t_{\Pi}}$ from $\pi_{I V}$, allowing in each case to find the optimal values $k, \pi_{I}, \pi_{I I}$ and $T_{1} / T_{3}$ for $\pi_{I V}$ data; 2) significant differences of this dependence from the dependence $\eta_{t_{k}}=f\left(T_{1}, T_{2}, \Delta T\right)$.

They show that the increase of economic efficiency is not enough to strive to increase the values of the polytropic exponent, since under certain conditions it can lead to the opposite result.

Analysis graphs below allows to choose the most advantageous values $\eta_{t_{\Pi}}$ and $\eta_{t_{k}}$ for each particular case, and hence the value of the coefficient of completeness of the reversible conversion of energy $\eta_{\mathrm{o}}$, as well as match $P T(k, R)$. The relative efficiency of actual cycles depending on the working bodies compared to the Carnot cycle efficiency can be estimated by the number of work.

\subsection{Development of a methodology of exergy analysis}

During the research, it was found that the exergy efficiency of the cryogenic cooling system is as follows:

$$
\eta_{\mathrm{e}}=\frac{\mathrm{Q}_{0} \cdot \tau_{\mathrm{e}}}{\mathrm{L}}
$$

where $Q_{0}-$ cooling capacity of the system; $\tau_{e}-$ exergy temperature function; $\mathrm{L}$ - cycle operation.

In this case, taking into account the costs for the preparation of the working fluid, exergy efficiency is determined by eqn:

$$
\eta_{\text {ep }}=\frac{\mathrm{Q}_{0} \cdot \tau_{\mathrm{e}}}{\sum \mathrm{E}+\mathrm{L}}
$$

where $\sum E$ - total energy input.

The total exergy efficiency, taking into account the costs for the preparation of the working fluid, as well as the costs for cooling system transportation on the power plant is determined by eqn:

$$
\eta_{\mathrm{et}}=\frac{\mathrm{Q}_{0} \cdot \tau_{\mathrm{e}}}{\sum \mathrm{E}+\mathrm{L}+\mathrm{e}_{\mathrm{m}}}
$$

where $\mathrm{e}_{\mathrm{m}}-$ exergy mass ratio.

$$
\mathrm{e}=\mathrm{k}_{\mathrm{m}} \cdot \mathrm{m}_{\mathrm{cs}}
$$

where $\mathrm{k}_{\mathrm{m}}$ - mass ratio, $\mathrm{m}_{\mathrm{cs}}-$ mass cooling system.

$$
\mathrm{k}_{\mathrm{m}}=\frac{\mathrm{m}_{\mathrm{f}} \cdot \mathrm{H}}{\mathrm{m}_{\mathrm{pp}} \cdot \mathrm{V}}
$$

where $\mathrm{m}_{\mathrm{f}}-$ fuel mass; $\mathrm{m}_{\mathrm{pp}}-$ power plant mass; $\mathrm{H}-$ combustion value; $\mathrm{V}$ - power plant work time.

The mass ratio $\mathrm{k}_{\mathrm{m}}$ and the exergy mass ratio $\mathrm{e}_{\mathrm{m}}$ are the new values that need to be taken into account in the calculations.

\section{Conclusions}

The result of this study is a new universal method of exergy analysis, which can be used to select a cryogenic cooling system. Applying the obtained methodology allows us to perform comprehensive analysis of the cryogenic cooling systems effectiveness. This, in turn, allows us to choose the most effective cooling system and to measure the performance of power plants in the industry, aviation, mechanical engineering. In addition, 
this technique allows to evaluate the effectiveness of the special measuring equipment.

\section{References}

1. Dovgyallo A.I., Nekrasova S.O., Sarmin D.V., Uglanov D.A. Imitations tests of throttle cooling system with cryogenic refueling tank and its comparative characteristics // Applied Physics. 2013. Issue 4, 2013, Pages. P. 54-59

2. Samad Jafarmadar, Peyman Nemati. Exergy analysis of diesel/biodiesel combustion in a homogenous charge compression ignition (HCCI) engine using three-dimensional model // Renewable Energy (V.9). 2016. Pages 514-523

3. Biryuk V.V., Tsapkova A. B., Shimanov A.A. Numerical modeling of cyclone machine for cleaning gas generator gases, Key Engineering Materials. 2016. Volume 685. Pages 153-157

4. V.A.F. Costa. On the exergy balance equation and the exergy destruction // Energy (V.116). Pages 824835

5. Nekrasova, S.O., Uglanov, D.A.,Shimanov, A.A. Comparative analysis of calculation models of pulse tube thermoacoustic refrigerator, 4th International Conference for Young Scientists High Technology: Research and Applications, HTRA 2015, 21 - 24 April 2015, Publisher: Trans Tech Publications Ltd, 2016 г., P. 3-7

6. Biryuk V.V., Gorshkalev A.A., Kayukov S.S., Uglanov D.A. Gas-Dynamic Analysis of Processes in a Small-Sizes Two-Stroke Combustion Engine // The Open Mechanical Engineering Journal. 2014. 8. P. 441-444 